\title{
Interval Analytic Method in Existence Result for Hyperbolic Partial Differential Equation
}

\author{
Peter 0. Arawomo \\ Department of Mathematics, University of Ibadan, Ibadan, Nigeria \\ Email: womopeter@gmail.com
}

Received 2 January 2014; revised 2 February 2014; accepted 15 February 2014

Copyright (C) 2014 by author and Scientific Research Publishing Inc.

This work is licensed under the Creative Commons Attribution International License (CC BY). http://creativecommons.org/licenses/by/4.0/

(c) (i) Open Access

\section{Abstract}

Without the usual assumption of monotonicity, we establish some results on the theory of hyperbolic differential inequalities which enable us to produce a majorising interval function for the solution of the hyperbolic initial value problem. Using this function, a variation of parameters formula and interval iterative technique, the existence of solution to the problem is established.

\section{Keywords}

\section{Interval Functions, Interval Majorant, Interval Extension, Interval Operator, Nested Sequence}

\section{Introduction}

In this paper, we utilize interval analytic methods in the investigation of the existence of solution of the hyperbolic partial differential equation

$$
z_{x y}=f\left(x, y, z, z_{x}, z_{y}\right),(x, y) \in I_{a b}
$$

with characteristic initial values

$$
\left.\begin{array}{l}
z(x, 0)=\sigma(x) ; x \in I_{a} . \\
z(0, y)=\tau(y) ; y \in I_{b} . \\
\sigma(0)=z(0,0)=\tau(0)=z_{0}
\end{array}\right\}
$$

prescribed in a two-dimensional rectangle $I_{a b}=I_{a} \times I_{b}$, where $I_{a}=[0, a], I_{b}=[0, b] \quad a, b \in \mathbb{R}$ and

$$
z_{x}=\frac{\partial z}{\partial x}, z_{y}=\frac{\partial z}{\partial y}, z_{x y}=\frac{\partial^{2} z}{\partial x \partial y}
$$


$f \in C\left(I_{a b} \times \mathbb{R}^{3}, \mathbb{R}\right), \quad \sigma \in C^{1}\left(I_{a}, \mathbb{R}\right), \quad \tau \in C^{1}\left(I_{b}, \mathbb{R}\right)$ and $z \in C^{1,2}\left(I_{a b}, \mathbb{R}\right)$ where $z \in C^{1,2}\left(I_{a b}, \mathbb{R}\right)$ means that $z$ is continuous on $I_{a b}$ and possesses continuous partial derivatives $z_{x}, z_{y}, z_{x y}$ on $I_{a b}$.

Without the assumption of monotonicity on the function $f$ we establish some results on the theory of hyperbolic differential inequalities which enable us to produce a majorizing interval function for the solution of the equation. With the use of a variation of parameters formula used in [1] and theorem 5.7 of [2] on interval iterative technique we generate a nested sequence of interval functions which converges to an interval solution. This interval solution is thus a majorant of the solution of the equation and it coincides with the real valued solution if it is degenerate. Similar interval methods had earlier been used by some authors in [3]-[7] for solution to differential equation but not for hyperbolic initial value problems. The result in this paper generalizes those of [1] [8] as the monotonicity condition imposed on the function $f$ is not in any way necessary.

The basic results in interval analysis used in this work are found in [2] [6] [7] [9]-[13] for readers who may not be familiar with them.

\section{Differential Inequalities and Majorisation of Solution}

Definition 2.1: A function $v \in C^{1,2}\left(I_{a, b}, \mathbb{R}\right)$ is said to be an upper solution of the hyperbolic initial value problem (1.1) and (1.2) on $I_{a b}$ if

$$
\begin{gathered}
v_{x y} \geq f\left(x, y, v, v_{x}, v_{y}\right), \quad(x, y) \in I_{a b} \\
v_{x}(x, 0) \geq \sigma^{\prime}(x), \quad x \in I_{a}, \\
v_{y}(0, y) \geq \tau^{\prime}(y), \quad y \in I_{b}, \\
v(0,0) \geq z_{0} .
\end{gathered}
$$

Definition 2.2: A function $u \in C^{1,2}\left(I_{a, b}, \mathbb{R}\right)$ is said to be a lower solution of the hyperbolic initial value problem (1.1) and (1.2) on $I_{a b}$ if the reversed inequalities hold true with $u$ in place of $v$ in the specified intervals.

Next, we shall consider some results concerning the upper and lower solutions of Equation (1.1) and conditions (1.2).

Theorem 2.1: Suppose that $u \in C^{1,2}\left(I_{a, b}, \mathbb{R}\right)$ and

$$
\left.\begin{array}{c}
u_{x y} \leq f\left(x, y, u, u_{x}, u_{y}\right), \\
v_{x y} \leq f\left(x, y, v, v_{x}, v_{y}\right), \\
u(0,0) \leq v(0,0) \\
u_{x}(x, 0) \leq v_{x}(x, 0), \quad x \in I_{b} . \\
u_{y}(0, y) \leq v_{y}(0, y), \quad y \in I_{b} .
\end{array}\right\}
$$

Then we have

$$
\left(u, u_{x}, u_{y}\right) \leq\left(v, v_{x}, v_{y}\right) \text { on } I_{a b}
$$

where the inequality is componentwise.

Proof: We shall establish this theorem by contradiction. From assumption (2.3) we see clearly that the theorem is true for the point $(0,0)$ on $I_{a b}$.

Suppose that inequality (2.4) is not true at a point $\left(x_{0}, y_{0}\right) \in I_{a b}$ and assume that

$$
u\left(x_{0}, y_{0}\right)>v\left(x_{0}, y_{0}\right) \text {, say, }
$$

then by assumption (2.3) $x_{0}$ and $y_{0}$ cannot both be zero.

Let $h>0$ be such that $\left(x_{0}, y_{0}+h\right) \in I_{a b}$, then $u\left(x_{0}, y_{0}+h\right) \leq v\left(x_{0}, y_{0}+h\right)$ and so

$$
u_{y}\left(x_{0}, y_{0}\right)=\lim _{h \rightarrow 0}\left\{\frac{u\left(x_{0}, y_{0}+h\right)-u\left(x_{0}, y_{0}\right)}{h}\right\}<\lim _{h \rightarrow 0}\left\{\frac{v\left(x_{0}, y_{0}+h\right)-v\left(x_{0}, y_{0}\right)}{h}\right\}=v_{y}\left(x_{0}, y_{0}\right)
$$


Thus, we have, for $y_{0} \neq 0$ (or $x_{0} \neq 0$ ),

$$
u\left(x_{0}, y_{0}\right)=u\left(x_{0}, 0\right)+\int_{0}^{y_{0}} u_{y}\left(x_{0}, t\right) \mathrm{d} t \leq v\left(x_{0}, 0\right)+\int_{0}^{y_{0}} v_{y}\left(x_{0}, t\right) \mathrm{d} t=v_{y}\left(x_{0}, y_{0}\right)
$$

and this contradicts assumption (2.5).

If $y_{0}=0$, then $x_{0} \neq 0$ (or vice-versa) and for $x_{0} \neq 0$ we have

$$
\begin{aligned}
u\left(x_{0}, y_{0}\right) & =u\left(x_{0}, 0\right)=u(0,0)+\int_{0}^{x_{0}} u_{x}(s, 0) \mathrm{d} s \\
& \leq v(0,0)+\int_{0}^{x_{0}} v_{x}(s, 0) \mathrm{d} s \quad \text { by condition }(2.3) \\
& =v\left(x_{0}, 0\right)
\end{aligned}
$$

If $y_{0} \neq 0$ and $x_{0}=0$ a similar argument can be advanced to obtain $u\left(0, y_{0}\right) \leq v\left(0, y_{0}\right)$. Hence,

$$
u\left(x_{0}, y_{0}\right) \leq v\left(x_{0}, y_{0}\right),
$$

and this is still a contradiction to our earlier assumption (2.5).

Suppose instead that

$$
u_{x}\left(x_{0}, y_{0}\right)>v_{x}\left(x_{0}, y_{0}\right) \text {, }
$$

Then $y_{0} \neq 0$, otherwise condition (2.3) would immediately give the required contradiction.

Now for $y_{0} \neq 0$, let $h>0$ such that $\left(x_{0}, y_{0}+h\right) \in I_{a b}$, we have $u_{x}\left(x_{0}, y_{0}+h\right) \leq v_{x}\left(x_{0}, y_{0}+h\right)$, so

$$
\begin{gathered}
u_{x y}\left(x_{0}, y_{0}\right)=\lim _{h \rightarrow 0}\left\{\frac{u_{x}\left(x_{0}, y_{0}+h\right)-u_{x}\left(x_{0}, y_{0}\right)}{h}\right\} \leq \lim _{h \rightarrow 0}\left\{\frac{v_{x}\left(x_{0}, y_{0}+h\right)-v_{x}\left(x_{0}, y_{0}\right)}{h}\right\}=v_{x y}\left(x_{0}, y_{0}\right) \\
\therefore u_{x}\left(x_{0}, y_{0}\right)=u_{x}\left(x_{0}, 0\right)+\int_{0}^{y_{0}} u_{x y}\left(x_{0}, t\right) \mathrm{d} t \\
\leq v_{x}\left(x_{0}, 0\right)+\int_{0}^{y_{0}} v_{x y}\left(x_{0}, t\right) \mathrm{d} s \quad \text { by condition }(2.3) \\
=v_{x}\left(x_{0}, y_{0}\right)
\end{gathered}
$$

This contradicts assumptions (2.6).

Similarly, if we assume that $u_{y}\left(x_{0}, y_{0}\right)>v_{y}\left(x_{0}, y_{0}\right)$, we would also arrive at a contradiction. At $x_{0}=a$ and $y_{0}=b$ left hand derivatives are used to obtain the result.

Hence, we conclude that, the assertion (2.4) holds true on $I_{a b}$ and this proves the theorem.

Theorem 2.2: Let $u$ and $v$ be functions defined on $I_{a b}$ which satisfy assumptions (2.1), (2.2) and (2.3) of Theorem 2.1. Suppose in addition that they satisfy the following conditions,

$$
\left.\begin{array}{l}
z_{0} \in[u(0,0), v(0,0)] \\
\sigma^{\prime}(x) \in\left[u_{x}(x, 0), v_{x}(x, 0)\right] ; \quad x \in I_{a} \\
\tau^{\prime}(y) \in\left[u_{y}(0, y), v_{y}(0, y)\right] ; \quad y \in I_{b}
\end{array}\right\}
$$

Then the solution $z$ of problem (1.1) and (1.2) together with its derivatives $z_{x}, z_{y}$ satisfy

$$
\left(z, z_{x}, z_{y}\right) \in\left([u, v],\left[u_{x}, v_{x}\right],\left[u_{y}, v_{y}\right]\right)
$$

On the rectangle, $I_{a b}$, where the inclusion is componentwise.

Proof: Notice that the lower endpoints of the intervals in Equation (2.7) satisfy assumption (2.3) of Theorem 2.1 when $v$ is replaced by $z$. Therefore $u$ and $z$ satisfy the hypothesis of Theorem 2.1 and hence

$$
\left(u, u_{x}, u_{y}\right) \leq\left(z, z_{x}, z_{y}\right) \text {, on } I_{a b}
$$


Similarly, replacing $u$ by $z$ in assumption (2.3) we obtain the upper endpoints of the intervals in conditions (2.7) and so by Theorem 2.1 we also have

$$
\left(z, z_{x}, z_{y}\right) \leq\left(v, v_{x}, v_{y}\right)
$$

Combining inequalities (2.8) and (2.9) we have the desired result.

\section{Construction and Existence of Solution}

Our purpose in this section is to establish the existence of solution to the problem (1.1) satisfying initial values (1.2) by means of interval analytic method. To this end an integral operator is constructed, the solution of the resulting operator equation is equivalent to the solution of the initial value problem under consideration. An interval extension of this operator is then used to generate a sequence of interval functions which converges to the required solution.

Let $z \in C^{1,2}\left(I_{a b}, \mathbb{R}\right)$ be such that $\left(u, u_{x}, u_{y}\right) \leq\left(z, z_{x}, z_{y}\right) \leq\left(v, v_{x}, v_{y}\right)$ on $I_{a b}$ and a function $\varphi: I_{a b} \times \mathbb{R}^{3} \rightarrow \mathbb{R}$, defined by

$$
\varphi\left(x, y, z, z_{x}, z_{y}\right)=f\left(x, y, z, z_{x}, z_{y}\right)+\lambda^{2} z-\lambda z_{x}-\lambda z_{y}
$$

where $f$ is the function in Equation (1.1) and $\lambda \geq 0$ is a constant suitably chosen such that $\varphi \geq 0$. Clearly it can be seen that $\varphi$ is continuous on $I_{a b} \times \mathbb{R}^{3}$.

With this new function $\varphi$, Equation (1.1) becomes

$$
z_{x y}=\varphi\left(x, y, z, z_{x}, z_{y}\right)-\lambda^{2} z+\lambda z_{x}+\lambda z_{y}
$$

By using the variation of constant formula of Lemma 4.1 in [1], we obtain the solution of Equation (3.2), satisfying initial values (1.2) as:

$$
z(x, y)=\sigma(x) \mathrm{e}^{\lambda y}+\tau(y) \mathrm{e}^{\lambda x}-z_{0} \mathrm{e}^{\lambda(x+y)}+\mathrm{e}^{\lambda(x+y)} \int_{0}^{y} \int_{0}^{x} \varphi\left(s, t, z, z_{x}, z_{y}\right) \mathrm{e}^{-\lambda(s+t)} \mathrm{d} s \mathrm{~d} t
$$

Differentiating with respect to $x$, we obtain

$$
z_{x}(z, y)=\lambda z(x, y)+\mathrm{e}^{\lambda y}\left\{\sigma^{\prime}(x)-\lambda \sigma(x)+\int_{0}^{y} \varphi\left(x, t, z, z_{x}, z_{y}\right) \mathrm{e}^{-\lambda t} \mathrm{~d} t\right\}
$$

and similarly by differentiating with respect to $y$ we obtain

$$
z_{y}(x, y)=\lambda z(x, y)+\mathrm{e}^{\lambda x}\left\{\tau^{\prime}(y)-\lambda \tau(y)+\int_{0}^{x} \varphi\left(s, y, z, z_{x}, z_{y}\right) \mathrm{e}^{-\lambda s} \mathrm{~d} s\right\}
$$

Eliminating the derivatives $z_{x}$ and $z_{y}$ by introducing the function $z^{*}(x, y)=z_{x}(x, y)$ and $z^{* *}(x, y)=z_{y}(x, y)$ into the integro-differential equations we obtain the system of integral equations

$$
\begin{gathered}
z(x, y)=\sigma(x) \mathrm{e}^{\lambda y}+\tau(y) \mathrm{e}^{\lambda x}+z_{0} \mathrm{e}^{\lambda(x+y)}+\mathrm{e}^{\lambda(x+y)} \int_{0}^{y} \int_{0}^{x} \varphi\left(s, t, z, z^{*}, z^{* *}\right) \mathrm{e}^{-\lambda(s+t)} \mathrm{d} s \mathrm{~d} t \\
z^{*}(x, y)=\lambda z(x, y)+\mathrm{e}^{\lambda y}\left\{\sigma^{\prime}(x)-\lambda \sigma(x)+\int_{0}^{y} \varphi\left(x, t, z, z^{*}, z^{* *}\right) \mathrm{e}^{-\lambda t} \mathrm{~d} t\right\} \\
z^{* *}(x, y)=\lambda z(x, y)+\mathrm{e}^{\lambda x}\left\{\tau^{\prime}(y)-\lambda \tau(y)+\int_{0}^{x} \varphi\left(s, y, z, z^{*}, z^{* *}\right) \mathrm{e}^{-\lambda s} \mathrm{~d} s\right\}
\end{gathered}
$$

which is equivalent to the problem (3.2) and initial values (1.2).

Denoting the right hand side of these integral equations by $p z, p^{*} z$ and $p^{* *} z$ respectively, we have the following:

$$
\left.\begin{array}{l}
z(x, y)=p z(x, y) \\
z^{*}(x, y)=p^{*} z(x, y) \\
z^{* * *}(x, y)=p^{* * *} z(x, y)
\end{array}\right\}
$$

With these we prove the following result. 
Lemma 3.1: Let $u$ and $v \in C^{1,2}\left(I_{a b}, \mathbb{R}\right)$ satisfy conditions (2.7) of Theorem 2.2. Suppose that for functions $\gamma \in C^{1,2}\left(I_{a b}, \mathbb{R}\right)$ with $\left(u, u_{x}, u_{y}\right) \quad\left(\gamma, \gamma_{x}, \gamma_{y}\right) \leq\left(v, v_{x}, v_{y}\right)$ on $I_{a b}$, we have

$$
\left.\begin{array}{l}
u_{x y} \leq f\left(x, y, \gamma, \gamma_{x}, \gamma_{y}\right)-\lambda^{2}(u-\gamma)+\lambda\left(u_{x}-\gamma_{x}\right)+\lambda\left(u_{y}-\gamma_{y}\right) \\
v_{x y} \geq f\left(x, y, \gamma, \gamma_{x}, \gamma_{y}\right)-\lambda^{2}(v-\gamma)+\lambda\left(v_{x}-\gamma_{x}\right)+\lambda\left(v_{y}-\gamma_{y}\right)
\end{array}\right\}
$$

where $\lambda$ is the constant appearing in Equation (3.1). Then the following hold true.

$$
\left.\begin{array}{l}
p \lambda(x, y) \in[u(x, y), v(x, y)] \\
p^{*} \gamma(x, y) \in\left[u^{*}(x, y), v^{*}(x, y)\right] \\
p^{* *}(x, y) \in\left[u^{* *}(x, y), v^{* *}(x, y)\right]
\end{array}\right\}
$$

for all $(x, y) \in I_{a b}$.

Proof: We first consider the lower endpoints of the inclusions and differentiating we have, from Equation (3.3)

$$
(p \gamma)_{x}=\lambda p \gamma+\mathrm{e}^{\lambda y}\left\{\sigma^{\prime}(x)-\lambda \sigma(x)+\int_{0}^{y} \varphi\left(x, t, \gamma, \gamma^{*}, \gamma^{* *}\right) \mathrm{e}^{-\lambda t} \mathrm{~d} t\right\}
$$

differentiating again with respect to $y$ we obtain

$$
\begin{aligned}
(p \gamma)_{x y} & =\lambda(p \gamma)_{y}+\lambda \mathrm{e}^{\lambda y}\left\{\sigma^{\prime}(x)-\lambda \sigma(x)+\int_{0}^{y} \varphi\left(x, t, \gamma, \gamma^{*}, \gamma^{* *}\right) \mathrm{e}^{-\lambda t} \mathrm{~d} t\right\}+\varphi\left(x, y, \gamma(x, y), \gamma^{*}(x, y), \gamma^{* *}(x, y)\right) \\
& =\lambda(p \gamma)_{y}+\lambda\left\{(p \gamma)_{x}-\lambda p \gamma\right\}+\varphi\left(x, y, \gamma, \gamma^{*}, \gamma^{* *}\right)
\end{aligned}
$$

This, by Equation (3.1) and assumption (3.7), gives

$$
(p \gamma-u)_{x y} \geq-\lambda^{2}(p \gamma-u)+\lambda(p \gamma-u)_{x}+\lambda(p \gamma-u)_{y} .
$$

Similarly by differentiating Equation (3.3) with respect to $y$, we obtain

$$
(p \gamma)_{y}=\lambda p \gamma+\mathrm{e}^{\lambda x}\left\{\tau^{\prime}(y)-\lambda \tau(x)+\int_{0}^{x} \varphi\left(s, y, \gamma(s, y), \gamma^{*}(s, y), \gamma^{* *}(s, y)\right) \mathrm{e}^{-\lambda s} \mathrm{~d} s\right\}
$$

By conditions (2.1) and (3.7) we have $(p \gamma-u)(0,0) \geq 0,(p \gamma-u)_{x}(x, 0) \geq 0$, for $x \in I_{a}$ and $(p \gamma-u)_{y}(0, y) \geq 0$ for $x \in I_{b}$. From these we see that $(p \gamma-u)$ satisfies the assumptions of Lemma 4.2 of [1] since $(p \gamma-u) \in C^{1,2}\left(I_{a b}, \mathbb{R}\right)$.

Thus

$$
\begin{gathered}
(p \gamma-u)(x, y) \geq 0 . \\
\Rightarrow u(x, y) \leq p \gamma(x, y), \quad(x, y) \in I_{a b}, \\
(p \gamma-u)_{x}(x, y) \geq 0 \\
\Rightarrow u^{*}(x, y) \leq p^{*} \gamma(x, y), \quad(x, y) \in I_{a b}, \\
(p \gamma-u)_{y}(x, y) \geq 0 \\
\Rightarrow u^{* *}(x, y) \leq p^{* *} \gamma(x, y), \text { for }(x, y) \in I_{a b},
\end{gathered}
$$

It could similarly be proved that

$$
\begin{gathered}
p \gamma(x, y) \leq v(x, y), \\
p^{*} \gamma(x, y) \leq v^{*}(x, y),
\end{gathered}
$$

and

$$
p^{* *} \gamma(x, y) \leq v^{* *}(x, y), \forall(x, y) \in I_{a b} .
$$


Hence the lemma is established.

Theorem 3.1: Let the functions $u, v \in C^{1,2}\left(I_{a b}, \mathbb{R}\right)$ satisfy conditions (2.7). Suppose that the function $f \in C^{1,2}\left(I_{a b} \times \mathbb{R}^{3}, \mathbb{R}\right)$ is such that

$$
u_{x y} \leq f\left(x, y, \gamma, \gamma_{x}, \gamma_{y}\right)-\lambda^{2}(u-\gamma)+\lambda\left(u_{x}-\gamma_{x}\right)+\lambda\left(u_{y}-\gamma_{y}\right)
$$

and

$$
v_{x y} \geq f\left(x, y, \gamma, \gamma_{x}, \gamma_{y}\right)-\lambda^{2}(v-\gamma)+\lambda\left(v_{x}-\gamma_{x}\right)+\lambda\left(v_{y}-\gamma_{y}\right),
$$

for function $\gamma \in C^{1,2}\left(I_{a b}, \mathbb{R}\right)$ satisfying

$$
\left(u, u_{x}, u_{y}\right) \leq\left(\gamma, \gamma_{x}, \gamma_{y}\right) \leq\left(v, v_{x}, v_{y}\right) \text { on } I_{a b} .
$$

and $\lambda \geq 0$, constant, suitably chosen in Equation (3.1).

Then there exists a convergent nested sequence of interval functions $\left\{Z_{n}\right\}$ such that the unique solution $z$ of Equations (1.1) and (1.2) satisfies

$$
z(x, y) \in \lim _{n \rightarrow \infty} Z_{n}(x, y)=Z(x, y), \forall x, y \in I_{a b}
$$

with $z=Z, Z$ degenerate where the initial interval $Z_{0}$ is given by

$$
Z_{0}(x, y)=[u(x, y), v(x, y)] .
$$

Proof: From the construction earlier considered, we see that any solution of Equation (1.1) which satisfies condition (1.2) solves the integral Equation (3.3). Conversely if $z$ solves the integral Equation (3.3) we have that

$$
z_{x y}=\lambda(p z)_{x}+\lambda(p z)_{y}-\lambda^{2}(p z)+\varphi\left(x, y, z, z, z_{x}, z_{y}\right)
$$

which by Equations (3.1) and (3.6) gives

$$
z_{x y}=f\left(x, y, z, z_{x}, z_{y}\right)
$$

with

$$
z(0,0)=z_{0}, z(x, 0)=\sigma(x), z(0, y)=\tau(y) .
$$

and these imply that $z$ again solves the Equation (1.1) and satisfies condition (1.2). Therefore, we shall seek the solution of the integral equation given by (3.3) which is transformed to the operator equation

$$
z=p z \text {. }
$$

Let $Z$ be an interval function defined on $I_{a b}$, such that $z(x, y) \in Z(x, y)$ for $(x, y) \in I_{a b}$, and the interval function $\Phi$ an interval extension of the function $\varphi$ defined in Equation (3.1). Then the interval integral operator $P$ defined by

$$
P Z=\sigma(x) \mathrm{e}^{\lambda y}+\tau(y) \mathrm{e}^{\lambda x}-z_{0} \mathrm{e}^{\lambda(x+y)}+\mathrm{e}^{\lambda(x+y)} \int_{0}^{y} \int_{0}^{x} \Phi\left(s, t, Z(s, t), Z^{*}(s, t), Z^{* *}(s, t)\right) \mathrm{e}^{-\lambda(s+t)} \mathrm{d} s \mathrm{~d} t
$$

is an interval majorant of $p$.

Then the problem reduces to solving the interval operator equation

$$
Z=P Z \text {. }
$$

However to determine $Z$ we need to also determine $Z^{*}$ and $Z^{* *}$ which are respectively interval extensions to the function $z^{*}$ and $Z^{* * *}$. This is done by solving the interval operator equations

$$
Z^{*}=P^{*} Z \text {, and } Z^{* *}=P^{* *} Z
$$

With $P^{*}$ and $P^{* *}$ defined respectively by

$$
P^{*} Z=\lambda Z(x, y)+\mathrm{e}^{\lambda y}\left\{\sigma^{\prime}(x)-\lambda \sigma(x)+\int_{0}^{x} \Phi\left(x, t, Z(x, t), Z^{*}(x, t), Z^{* *}(x, t)\right) \mathrm{e}^{\lambda x} \mathrm{~d} t\right\}
$$

and 


$$
P^{* *} Z=\lambda Z(x, y)+\mathrm{e}^{\lambda x}\left\{\tau^{\prime}(y)-\lambda \tau(y)+\int_{0}^{x} \Phi\left(s, y, Z(s, y), Z^{*}(s, y), Z^{* *}(s, y)\right) \mathrm{e}^{\lambda s} \mathrm{~d} s\right\}
$$

which majorise the real operators $p^{*}$ and $p^{* *}$ respectively.

Define the sequences $\left\{Z_{n}\right\},\left\{Z_{n}^{*}\right\},\left\{Z_{n}^{* *}\right\}$ by

$$
\begin{gathered}
Z_{n+1}=P Z_{n}, n=0,1,2, \cdots \text { with } \\
Z_{0}=[u(x, y), v(x, y)], \\
Z_{n+1}^{*}=P^{*} Z_{n}, n=0,1,2, \cdots \text { with } \\
Z_{0}^{*}=\left[u^{*}(x, y), v^{*}(x, y)\right] \text { and } \\
Z_{n+1}^{* *}=P^{* *} Z_{n}, n=0,1,2, \cdots \text { with } \\
Z_{0}^{* *}=\left[u^{* *}(x, y), v^{* *}(x, y)\right]
\end{gathered}
$$

We have the sequence $\left\{Z_{n}\right\}$ as required.

We shall show that $\left\{Z_{n}\right\}$ convergences to a limit. But this can only be so if the sequence $\left\{Z_{n}^{*}\right\}$ and $\left\{Z_{n}^{* *}\right\}$ also converge.

By Theorem 5.7 of [2], these sequences converge if

$$
\begin{gathered}
P\left(Z_{0}\right) \subseteq Z_{0}, \\
P^{*}\left(Z_{0}^{*}\right) \subseteq Z_{0}^{*} \text { and } \\
P^{* *}\left(Z_{0}^{* *}\right) \subseteq Z_{0}^{* *}
\end{gathered}
$$

Now for

$$
\begin{gathered}
Z_{0}=[u(x, y), v(x, y)], \\
P\left(Z_{0}\right)=\{p z: z \in[u(x, y), v(x, y)]\} \subseteq[p u(x, y), p v(x, y)] \subseteq[u(x, y), v(x, y)]
\end{gathered}
$$

by the first inclusion of Equation (3.8). Hence

$$
P\left(Z_{0}\right) \subseteq Z_{0} .
$$

Similarly we have by the result given in Equation (3.8) of Lemma 3.1

$$
P^{*}\left(\left[u^{*}(x, y), v^{*}(x, y)\right]\right) \subseteq\left[u^{*}(x, y), v^{*}(x, y)\right]
$$

and

$$
P^{* *}\left(\left[u^{* *}(x, y), v^{* *}(x, y)\right]\right) \subseteq\left[u^{* *}(x, y), v^{* *}(x, y)\right]
$$

Since these initial intervals satisfy the hypothesis of Theorem 5.7 of [2], the result of the theorem implies that $Z^{*}, Z^{* *}$ and $Z$ converge as sequences and are equally nested. Furthermore, the solution $Z$ of Equation (1.1) satisfying condition (1.2) belongs to the limit function $Z$ of the sequence $\left\{Z_{n}\right\}$, that is,

$$
z(x, y) \in Z(x, y)=\lim _{n \rightarrow \infty} Z_{n}(x, y)=\bigcap_{n=0}^{\infty} Z_{n}(x, y)
$$

and this proves the theorem.

Lemma 3.2: Assume that the functions $u, v \in C^{1,2}\left(I_{a b}, \mathbb{R}\right)$ satisfy conditions (2.7) and in addition they also satisfy conditions (2.1) and (2.2). Suppose further that the function $f$ appearing on the right hand side of Equation (1.1) satisfies:

$$
f(x, y, \alpha, \beta, \gamma)-f\left(x, y, \alpha_{1}, \beta_{1}, \gamma_{1}\right) \geq-\lambda^{2}\left(\alpha-\alpha_{1}\right)+\lambda\left(\beta-\beta_{1}\right)+\lambda\left(\gamma-\gamma_{1}\right)
$$

whenever the functions $\gamma, \beta, \alpha, \gamma_{1}, \beta_{1}$ and $\alpha_{1}$ are such that 


$$
\left(u, u_{x}, u_{y}\right) \leq\left(\alpha_{1}, \beta_{1}, \gamma_{1}\right) \leq(\alpha, \beta, \gamma) \leq\left(v, v_{x}, v_{y}\right)
$$

for constant $\lambda \geq 0$, suitably chosen. Then we have

$$
\left.\begin{array}{l}
p \psi(x, y) \in[u(x, y), v(x, y)] \\
p^{*} \psi(x, y) \in\left[u^{*}(x, y), v^{*}(x, y)\right] \\
p^{* *} \psi(x, y) \in\left[u^{* *}(x, y), v^{* *}(x, y)\right]
\end{array}\right\}
$$

for any function $\psi$ satisfying

$$
\left(u, u_{x}, u_{y}\right) \leq\left(\psi, \psi_{x}, \psi_{y}\right) \leq\left(v, v_{x}, v_{y}\right),(x, y) \in I_{a b} .
$$

Proof: From inequality (2.1) we have

$$
-u_{x y} \geq-f\left(x, y, u, u_{x}, u_{y}\right)
$$

Since

$$
\left(u, u_{x}, u_{y}\right) \leq\left(\psi, \psi_{x}, \psi_{y}\right),
$$

From inequality (3.9) we have

$$
-\lambda^{2}(\psi-u)+\lambda\left(\psi_{x}-u_{x}\right)+\lambda\left(\psi_{y}-u_{y}\right) \leq f\left(x, y, \psi, \psi_{x}, \psi_{y}\right)-f\left(x, y, u, u_{x}, u_{y}\right) \leq f\left(x, y, \psi, \psi_{x}, \psi_{y}\right)-u_{x y}
$$

and so

$$
u_{x y} \leq f\left(x, y, \psi, \psi_{x}, \psi_{y}\right)-\lambda^{2}(u-\psi)+\lambda\left(u_{x}-\psi_{x}\right)+\lambda\left(u_{y}-\psi_{y}\right)
$$

which is the first inequality in (3.7).

Also from inequality (2.2) we have

$$
v_{x y} \geq f\left(x, y, v, v_{x}, v_{y}\right)
$$

and using inequality (3.9) we have

$$
-\lambda^{2}(v-\psi)+\lambda\left(v_{x}-\psi_{x}\right)+\lambda\left(v_{y}-\psi_{y}\right) \leq f\left(x, y, v, v_{x}, v_{y}\right)-f\left(x, y, \psi, \psi_{x}, \psi_{y}\right) \leq v_{x y}-f\left(x, y, \psi, \psi_{x}, \psi_{y}\right)
$$

Therefore

$$
v_{x y} \geq f\left(x, y, \psi, \psi_{x}, \psi_{y}\right)-\lambda^{2}(v-\psi)+\lambda\left(v_{x}-\psi_{x}\right)+\lambda\left(v_{y}-\psi_{y}\right)
$$

which also is the second inequality in (3.7). Since all the other conditions of Lemma 3.1 are also satisfied, the proof of this lemma follows as for Lemma 3.1 to obtain the desired result.

Remark 3.1: If $\lambda=0$ in inequality (3.9) then we have

$$
f(x, y, \alpha, \beta, \gamma)-f\left(x, y, \alpha_{1}, \beta_{1}, \gamma_{1}\right) \geq 0
$$

for $\left(\alpha_{1}, \beta_{1}, \gamma_{1}\right) \leq(\alpha, \beta, \gamma)$ and this implies that $f$ is monotone increasing in its domain of definition. Therefore the result of lemma 3.2 also holds for a monotone function $f$.

Theorem 3.2: Suppose that the function $u, v \in C^{1,2}\left(I_{a b}, \mathbb{R}\right),\left(u, u_{x}, u_{y}\right) \leq\left(v, v_{x}, v_{y}\right)$, satisfies conditions (2.1), (2.2) and (2.7). If in addition the function $f$ appearing in Equation (1.1) satisfies

$$
f(x, y, \alpha, \beta, \gamma)-f\left(x, y, \alpha_{1}, \beta_{1}, \gamma_{1}\right) \geq-\lambda^{2}\left(\alpha-\alpha_{1}\right)+\lambda\left(\beta-\beta_{1}\right)+\lambda\left(\gamma-\gamma_{1}\right)
$$

whenever

$$
\left(u, u_{x}, u_{y}\right) \leq\left(\alpha_{1}, \beta_{1}, \gamma_{1}\right) \leq(\alpha, \beta, \gamma) \leq\left(v, v_{x}, v_{y}\right) \text { on } I_{a b} .
$$

for some constant $\lambda \geq 0$, suitably chosen.

Then there exists a nested sequence of interval function $\left\{Z_{n}\right\}, n \in \mathbb{N}$ with each term majorising the unique solution $Z$ of Equation (1.1) satisfying condition (1.2) such that the limit $Z$ of this sequence also contains $Z$, 
that is,

$$
z(x, y) \in Z(x, y)=\lim _{n \rightarrow \infty} Z_{m}(x, y) .
$$

Proof: As it has been shown in the proof of Lemma 3.2, the conditions prescribed in this theorem can equally be linked with those of Theorem 3.1. Therefore the proof can be established in a manner similar to that of Theorem 3.1 .

\section{References}

[1] Lakshmikantham, V. and Pandit, S.G. (1985) The Method of Upper and Lower Solution and Hyperbolic Partial Differential Equations. Journal of Mathematical Analysis and Applications, 105, 466-477. http://dx.doi.org/10.1016/0022-247X(85)90062-9

[2] Moore, R.E. (1979) Methods and Application of Interval Analysis. SIAM (Studies in Applied and Numerical Mathematics), Philadelphia. http://dx.doi.org/10.1137/1.9781611970906

[3] Arawomo, P.O. and Akinyele, O. (2002) An Interval Analytic Method in Constructive Existence Result for Initial Value Problems. Dynamic Systems and Applications, 11, 545-556.

[4] Chan, C.Y. and Vatsala, A.S. (1990) Method of Upper and Lower Solution and Interval Method for Semilinear EulerPision-Darboux Equations. Journal of Mathematical Analysis and Applications, 150, 378-381. http://dx.doi.org/10.1016/0022-247X(90)90110-2

[5] Lakshmikantham, V. and Swansundaran, S. (1987) Interval Method for 1st Order Differential Equations. Applied Mathematics and Computation, 23, 1-5. http://dx.doi.org/10.1016/0096-3003(87)90052-X

[6] Moore, R.E. (1984) Upper and Lower Bounds on Solutions of Differential, Integral Equations without Using Monotonicity, Convexity or Maximum Principle., In: Vichnevetsky, R. and Stepheman, R., Eds., Advances in Computer Methods for Partial Differential Equations, IMACS, 458-461.

[7] Moore, R.E. (1984) A Survey of Interval Methods for Differential Equations. Proceedings of 23rd Conference on Decision and Control, Las Vegas, 12-14 December 1984, 1529-1535.

[8] Walter, W. (1970) Differential and Integral Inequalities. Springer-Verlag, Berlin, Heidelberg. http://dx.doi.org/10.1007/978-3-642-86405-6

[9] Caprani, O. and Madsen, K. (1975) Contraction Mappings in Interval Analysis. BIT Numerical Mathematics, 15, 362366. http://dx.doi.org/10.1007/BF01931673

[10] Moore, R.E. (1977) A Test for Existence of Solution to Nonlinear Systems. SIAM Journal on Numerical Analysis, 14, 611-615.

[11] Moore, R.E. (1978) Bounding Sets in Function Spaces with Application to Nonlinear Operator Equations. SIAM Review, 20, 492-512.

[12] Rall, L.B. (1983) Mean-Value and Taylor Forms in Interval Analysis. SIAM Journal on Mathematical Analysis, 14, 223-238.

[13] Rall, L.B. (1982) Integration of Interval Functions II, the Finite Case. SIAM Journal on Mathematical Analysis, 13, 690-698. 